\title{
Pathways to Social Inequality
}

Hannah J. Haynie ${ }^{1,2}$, Patrick H. Kavanagh ${ }^{1}$, Fiona M. Jordan ${ }^{3}$, Carol R. Ember ${ }^{4}$, Russell D. Gray ${ }^{5}$, Simon J. Greenhill ${ }^{5,6}$, Kathryn R. Kirby ${ }^{5,7}$, Geoff Kushnick ${ }^{8}$, Bobbi S. Low ${ }^{9}$, Ty Tuff ${ }^{10}$,

Bruno Vilela $^{11}$, Carlos A. Botero ${ }^{12}$, and Michael C. Gavin ${ }^{1,5}$

${ }^{1}$ Department of Human Dimensions of Natural Resources, Colorado State University, Fort Collins, CO, USA

${ }^{2}$ Department of Linguistics, University of Colorado, Boulder, CO, USA

${ }^{3}$ Department of Anthropology and Archaeology, University of Bristol, Bristol, United Kingdom

${ }^{4}$ Human Relations Area Files, Yale University, New Haven, CT, USA

${ }^{5}$ Department of Linguistic and Cultural Evolution, Max Planck Institute for The Science of Human History, Jena, Germany

${ }^{6}$ ARC Centre of Excellence for the Dynamics of Language, Australian National University, Canberra, ACT, Australia

${ }^{7}$ Department of Ecology and Evolutionary Biology, University of Toronto, Toronto, ON, Canada ${ }^{8}$ School of Archaeology and Anthropology, Australian National University, Canberra, ACT, Australia

${ }^{9}$ School of Natural Resources and Environment, University of Michigan, Ann Arbor, MI, USA

${ }^{10}$ Department of Biology, McGill University, Montreal, QC, Canada

${ }^{11}$ Institute of Biology, Universidade Federal da Bahia, Salvador, BA, Brazil

${ }^{12}$ Department of Biology, Washington University in St. Louis, St. Louis, MO, USA 
Social inequality is now pervasive in human societies, despite the fact that humans lived in rel-

atively egalitarian, small-scale societies across most of our history. Prior literature highlights the importance of environmental conditions, economic defensibility, and wealth transmission for shaping early Holocene origins of social inequality. However, it remains untested whether the mechanisms that drive the evolution of inequality in recent human societies follow a similar trajectory. We conduct the first global analysis of pathways to inequality within modern human societies using structural equation modeling. Our analytical approach demonstrates that environmental conditions, resource intensification, and wealth transmission mechanisms impact various forms of social inequality via a complex web of causality. We further find that subsistence practices have a direct impact on some institutionalized forms of inequality. This work identifies drivers of social inequality in the modern world and demonstrates the application of structural equation modeling methods to investigate complex relationships between elements of human culture.

\section{Introduction}

Social and economic inequality are ubiquitous in contemporary human societies, a trend that has been linked to a number of detrimental consequences for the environment, the stability of political and economic systems, and the well-being of individuals (e.g. 1; 2; 3). This inequality has been formalized and reinforced in well-documented societies by cultural institutions like social class hierarchies, caste systems, and slavery. However, human social organization is commonly characterized as having consisted of essentially egalitarian, small-scale societies for the majority of human history $(4 ; 5 ; 6)$. While both external and intentional leveling mechanisms may have con- 
tributed to the pervasiveness of egalitarian, small-scale social organization earlier in human history, in contrast to the strict social hierarchies common in other primate species (e.g. 7;8), a different set of mechanisms has been proposed to explain the emergence of social inequality and its widespread occurrence in modern cultures. Here we investigate institutionalized forms of inequality in modern human societies and potential causal relationships with a set of mechanisms that represent a possible trajectory for the evolution and maintenance of inequality in recent human history.

Literature on the evolution of social inequality has focused primarily on reasons for the rise of inequality around the dawn of the Holocene (e.g. 5; 6; 9). Somewhat less attention has been paid to the processes that have caused this element of human social organization to continue to emerge, persist, and evolve in societies during the intervening millennia of human history, or its occurrence in a diverse and geographically widespread range of modern human cultures. Our current understanding of the evolution of social inequality does not extend to whether mechanisms and pathways associated with the de novo origin of human social inequality might also shape the subsequent evolution and persistence of inequality in modern societies. We might expect facets of environmental suitability, investment in resources, and wealth transmission patterns to impact inequality in recently documented societies, just as they are hypothesized to shape the early evolution of inequality (10). We test one potential extension of a generalized pathway for the evolution of inequality to the generation and maintenance of three specific types of institutionalized inequality in modern societies, using cross-cultural data collected in the Ethnographic Atlas and linked to environmental information in the D-PLACE database $(11 ; 12 ; 13 ; 14 ; 15 ; 16)$.

The timing of the earliest evidence of human social inequality has been linked to patterns of 
climate change, and specifically a decline in climate variability around 12,000 years ago $(9 ; 17 ; 18)$. This ecological shift is theorized to have made risk mitigation strategies that previously leveled social hierarchies less necessary, changing the relationships between humans and natural resources in the process $(10 ; 19 ; 20 ; 21 ; 22)$. Though this sort of global shift in climatic conditions may have opened the door to incipient inequality at one specific point in human history, where conditions persist that make resources dense or predictable we might expect to find ongoing evidence for environmental impacts on the mechanisms that shape inequality. In particular, local environmental conditions may play an important role in the intensification of subsistence activities, a cultural shift that we expect to have consequences for the distribution and accumulation of wealth, and thus on the development of cultural institutions that reinforce inequality.

Economic defensibility has been ascribed a key role in the emergence of inequality in early Holocene small-scale societies. This principle of resource management entails a comparison between the costs of defending a resource patch through actions such as monitoring and preventing intruders, and the resulting benefits (23). Dense, predictable resources are more defensible, as they are associated with relatively small areas to defend, they are easy to locate and monitor, and the reliable and abundant resources they produce counter-balance the cost of defense. Behaviours and norms that are focused on the defense of natural resources, like territoriality and land ownership, are theorized to arise when their benefits outweigh their costs (24). In early Holocene human groups, the scales may have tipped toward the adoption of these resource defense strategies when increasingly stable environmental conditions led to highly reliable or concentrated resource patches (10). Over the subsequent millenia of cultural evolution, human innovations such as cultivation of agricultural resources and intensification of production have further enhanced the density and pre- 
dictability of resources, and thus their defensibility, in some societies (25). Though research on early Holocene origins of inequality focuses on defensibility, we note that the sort of extraordinary global climate shift that led to enhanced defensibility at the dawn of the Holocene has not happened since. Thus we hypothesize that the intensification of subsistence resources may play a particularly important role in shaping the use, defense, and distribution of resources. Rather than simply implementing proxies for a mechanism associated with early Holocene inequality, we predict that more intensive production technologies and the associated property ownership will lead to unequal accumulation of wealth, and ultimately a greater likelihood of institutionalized inequality.

Intergenerational transmission of wealth allows unequal distributions of resources in a society to accumulate and persist over time, and is widely believed to play a role in social inequality in both ancient and modern societies (e.g. 5; 26; 27). Wealth includes material assets like land and tangible property, as well as social wealth (e.g. support networks, power) and embodied wealth (e.g. physical health, knowledge) $(27 ; 28)$. Material wealth is hypothesized to be particularly closely linked to inequality $(25 ; 26 ; 29)$, especially in agricultural or pastoral societies $(25 ; 27)$. Inheritance of finite resources, like land, whose productivity does not generally increase rapidly without major environmental or cultural shifts, may magnify asymmetries in resource distribution, and thus may be particularly important to the genesis of inequality (25). Although unequal wealth itself in the form of property rights may emerge before agriculture (19), the impacts of wealth transmission on social mobility may play an important role in linking subsistence activities to institutionalization of inequality.

Though inequality can be operationalized in a number of ways, we focus on a small set of 
outcomes that represent rigid and persistent institionalization of inequality. Social stratification in general may be characterized by heritable differences in the level of access to resources and power enjoyed by members of a society. Some level of social and economic inequality may have existed throughout human history merely due to individual variation in economic success and differences in the resources controlled by different families or lineages (4). However, the persistent, institutionalized inequality that is the focus of this study occurs only when differences in resources and power become entrenched in a society's norms and practices to the extent that social status persists across generations. We focus on a narrow set of observable institutions which require widespread acceptance of persistent social hierarchies, are unequivocally associated with social inequality, and can be reliably coded as present or absent across a wide range of societies. We acknowledge that inequality takes many forms in human society, and that alternative characterizations of inequality (e.g. Gini coefficients) are important for understanding other dimensions of inequality. We propose that investigating a limited and cohesive set of variables across a global sample of societies provides a perspective not already captured in nation-level economic studies or detailed case studies of individual cultures.

Using this framework, we examine the extent to which empirical evidence supports the hypothesis that the evolution and maintenance of persistent, institutionalized social inequality in modern human societies follows a trajectory that parallels the pathway by which inequality is thought to have taken hold earlier in human history. Recent work has described the set of mechanisms involved in this pathway as causal links between environmental conditions, resource defensibility, wealth transmission, and inequality (10). We propose an analogous trajectory for more recent development and maintenance of inequality that focuses not on economic defensibility but on the 
intensification of subsistence practices and the role these practices play in shaping human use of environmental resources and the ownership and transmission of property. This trajectory can be schematized as a path model (Fig. 2a). We investigate the details of each of the theoretical constructs in this framework using a large, global cross-cultural data sample from the D-PLACE database of places, languages, culture, and environment (11), and examine both incremental and direct effects of the associated variables on institutionalized inequality by applying a structural equation model (SEM) approach.

In testing whether the factors linked to inequality in modern societies mirror the mechanisms proposed to explain the evolution of inequality in early Holocene cultures, this approach employs normative data at the society level to test predictions which are often derived from individual-level within-population phenomena. To the extent that we expect such phenomena to be detectable in population-level patterns, we see cross-cultural tests of behavioural ecology theory as an important source of evidence about these predictions (30). By taking advantage of the large global sample of society-level data for a complex set of variables, we are able to simultaneously consider multiple facets of the generalized pathways and specific associations identified in prior literature (e.g. 10; $28 ; 31)$.

\section{Results}

Our study compares three alternative models, which vary the number of variables and paths included on a standardized structure that reflects the general sequence and directionality of causal links proposed in prior literature to explain the emergence of inequality (10). In each path model 


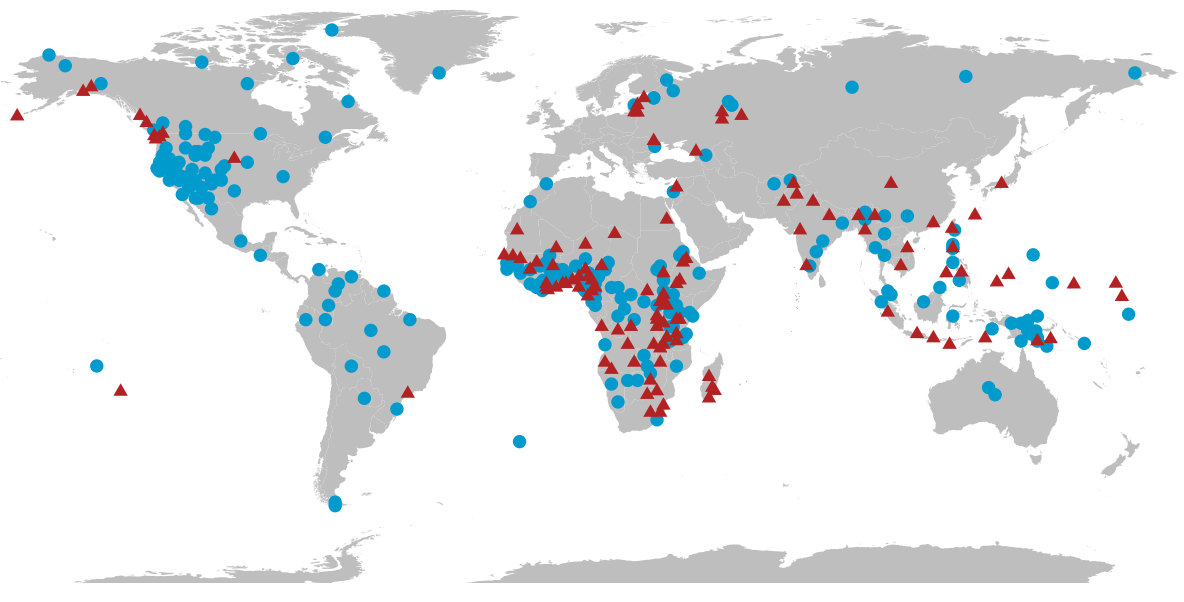

Figure 1: Societies included in the study $(n=367)$. Red triangles represent societies that are identified as having heritable social class systems. Blue dots represent societies with an absence of heritable social class.

the direction of causality in relationships between variables is assumed to follow a trajectory from environmental conditions to resource intensification to wealth transmission, resulting finally in inequality. Across these three models we vary only the inclusion of a population variable and the presence of individual pathways. The first alternative (Fig. 2b) restricts the paths in the model to a stepwise trajectory that includes only those direct effects that represent sequential links between constructs in the aforementioned order, with no additional direct paths (Fig. 2a). The second, more elaborate model (Fig. 2c) includes all potential direct effects whose directionality is consistent with the overall trajectory of the schema, as well as possible effects of population. A third model (Fig. 3) preserves well supported paths in Fig. 2c, but eliminates poorly supported paths, creating a more parsimonious model to fit these data. 
a.
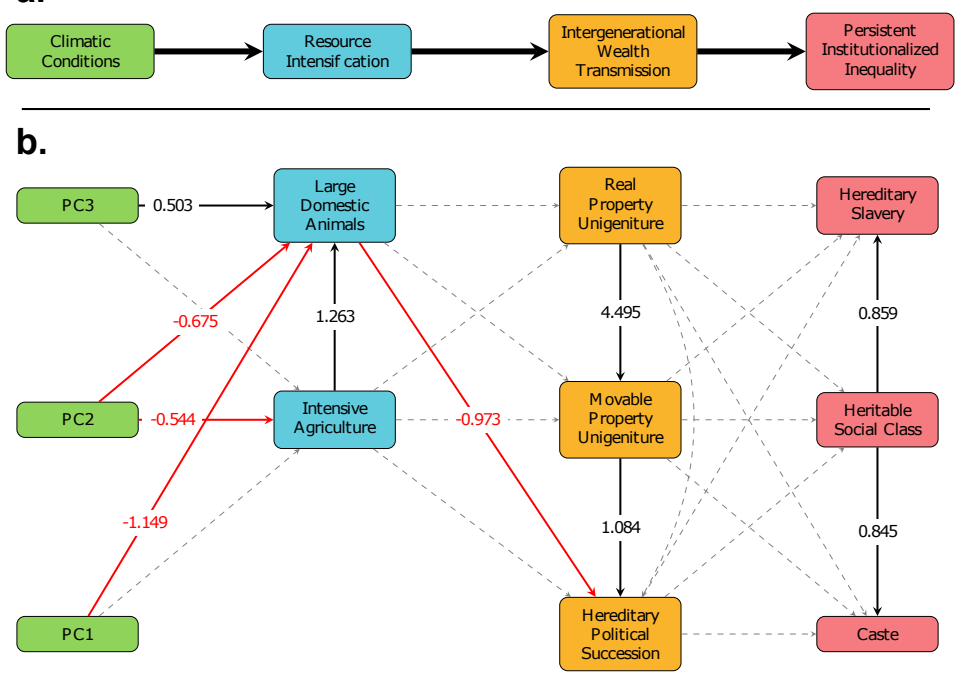

c.

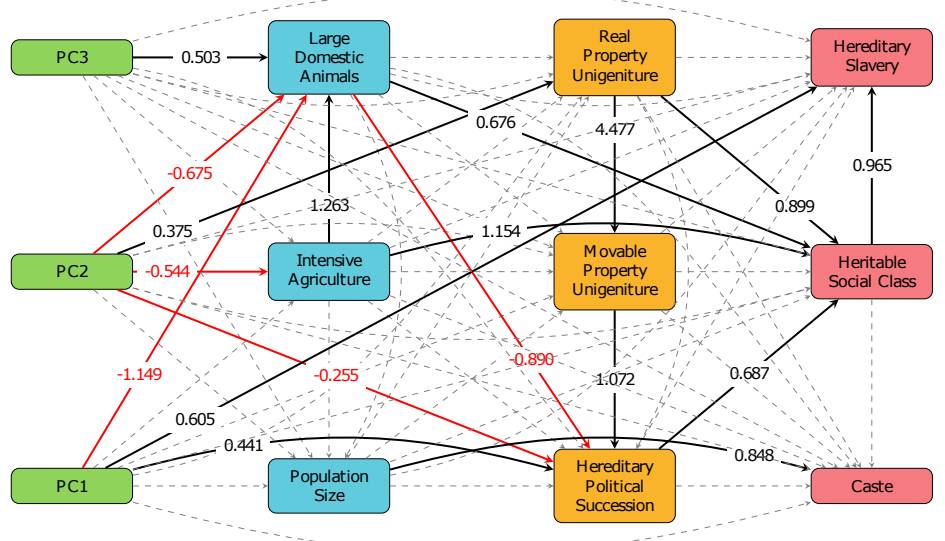

Figure 2: Path diagrams representing a) generalized trajectory, b) empirical SEM test of mechanisms on a strictly stepwise trajectory, interpreted through variables derived from DPLACE, and c) loose implementation of the hypothesized pathways using variables from D-PLACE, in which a population size variable and additional direct paths that deviate from the discrete steps in the schema have been included. Red arrows indicate negative relationships identified in PiecewiseSEM model. Black arrows represent positive relationships identified in PiecewiseSEM model. Dashed arrows represent paths not found to be significant $(\mathrm{p}<0.05)$. Significant paths are labeled with standardized coefficients. Individual variables represented by boxes in the diagram can be interpreted as increasing for continuous variables and present for categorical variables. See Methods for interpretation of PCA-derived environmental variables. Model $2 \mathrm{c}$ is better supported by the data than $2 \mathrm{~b}$ (AIC: Model 2b = 185.51, Model 2c = 162.48). $\mathrm{n}=367$. 


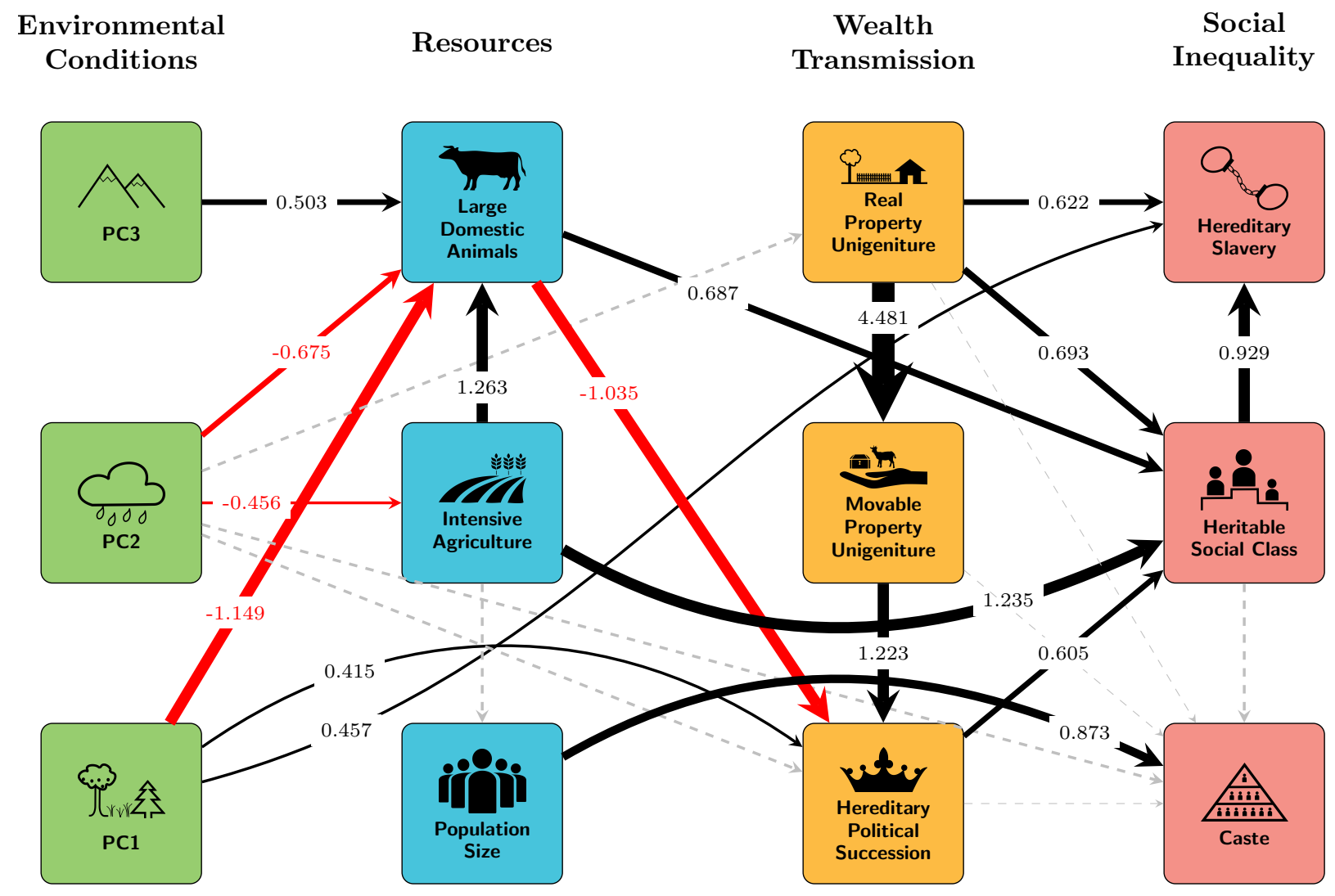

Figure 3: Most parsimonious model identified by Piecewise SEM analysis. Black arrows represent positive relationships. Red arrows represent negative relationships. Dashed arrows represent paths not found to be significant $(\mathrm{p}<0.05$ ) Line weights indicate the estimated magnitude of effects, and paths are labeled with standardized coefficients. Individual variables represented by boxes in the diagram can be interpreted as increasing for continuous variables and present for categorical variables. This model is better supported by the data than $2 b$ or $2 c$ (AIC: Model $2 b=185.51$, Model $2 \mathrm{c}=162.48$, Model $3=150.30$ ). $\mathrm{n}=367$. 
Shipley's test for directional separation indicates that paths are missing from the model that implements a strictly stepwise progression of mechanisms on the pathway to inequality (Fig. 2a, Fisher's $\mathrm{C}=99.51, \mathrm{df}=50, \mathrm{p}<0.0001)(32)$. Akaike Information Criterion comparison also suggests that the highly restricted path model in Fig. $2 \mathrm{~b}(\triangle \mathrm{AIC}=35.21, \mathrm{AIC}=185.51)$ does not account for the data as well as the more elaborate model in Fig. 2c $(\triangle \mathrm{AIC}=12.18, \mathrm{AIC}=162.48)$. The third model (Fig. 3) eliminates non-significant paths that are not identified as missing by Shipley's test and reduces unnecessary model complexity $(\mathrm{AIC}=150.30)$. AIC comparison suggests that of the models under consideration, this final model provides the best characterization of the relationships between variables in our dataset.

The model in Fig. 3 includes both direct and indirect effects of subsistence variables on inequality outcomes. Six pairs of variables are linked by both direct and indirect effects, including the links from intensive agriculture, large domesticated animals, and real property unigeniture to heritable social class that are hypothesized to be particularly important pathways. Table 1 provides a comparison between the direct effect and net indirect effect of each predictor on every inequality variable.

We find that the environment has important impacts on the activities that relate to resource intensification, namely intensive agriculture and keeping large domesticated animals. Environments with less seasonal climate variation are more likely to be associated with these two variables, both of which represent subsistence activities that may increase resource defensibility. We also find a direct link between environmental productivity and slavery, with hereditary slavery more likely to occur in more productive environments. Although this relationship is not mediated by the other 
Table 1: Comparison of the direct and indirect effects in structural equation model in Fig. 3 (standardized coefficients). Net indirect effects are calculated by multiplying coefficients along each indirect path that connects the predictor and the ultimate response and computing the sum of all indirect paths between predictor and response. For relationships that are comprised of both direct and indirect effects, bold text indicates the effect of greater magnitude.

\begin{tabular}{llrr}
\hline Response & Predictor & Direct & Indirect \\
\hline Caste & Population Size & 0.873 & \\
\hline Class & Intensive Agriculture & $\mathbf{1 . 2 3 5}$ & 0.077 \\
\hline Class & Large Domesticated Animals & $\mathbf{0 . 6 8 7}$ & -0.626 \\
\hline Class & Movable Property Unigeniture & & 0.739 \\
\hline Class & PC2 & & -0.640 \\
\hline Class & PC1 & 1.689 \\
\hline Class & Real Property Unigeniture & 0.693 & $\mathbf{3 . 3 1 2}$ \\
\hline Class & Hereditary Political Succession & 0.605 & \\
\hline Class & PC3 & & 0.031 \\
\hline Slavery & Intensive Agriculture & & 2.182 \\
\hline Slavery & Large Domesticated Animals & & 0.101 \\
\hline Slavery & Heritable Social Class & 0.929 & \\
\hline Slavery & Movable Property Unigeniture & & 0.687 \\
\hline Slavery & PC2 & & 2.120 \\
\hline Slavery & PC1 & & 0.168 \\
\hline Slavery & Real Property Unigeniture & 0.622 & $\mathbf{3 . 7 2 0}$ \\
\hline Slavery & Hereditary Political Succession & & 0.561 \\
\hline Slavery & PC3 & & 0.028 \\
\hline Succession & Intensive Agriculture & & -1.308 \\
\hline Succession & Large Domesticated Animals & -1.035 & \\
\hline Succession & Movable Property Unigeniture & 1.223 & \\
\hline Succession & PC2 & & 1.296 \\
\hline Succession & PC1 & & $\mathbf{1 . 1 8 9}$ \\
\hline Succession & Real Property Unigeniture & & \\
\hline Succession & PC3 & & \\
\hline & & & \\
\hline
\end{tabular}


cultural variables in our study, it is likely cultural in nature, reflecting the use of slave labor in exploiting abundant resources, or potentially a more complex cascade of economic and political developments arising from resource surplus. Though the role of environmental conditions in the early origins of inequality has been characterized primarily in terms of large-scale patterns in climate stability that created better conditions for resource defense in the early Holocene (22), we find that climatic predictability and environmental productivity may play complex roles in determining strategies for subsistence, shaping social and political processes, and even directly influencing the cultural institutions that formalize and reinforce inequality.

Our results also include strong evidence that wealth transmission norms, and particularly those that concentrate power and real property holdings, are associated with the institutionalization of social inequality through class systems in which social status is inherited. The association of both real property unigeniture and hereditary political succession with heritable social class supports the hypothesis that many types of wealth contribute to the generation of inequality $(10 ; 27 ; 28)$. Of the wealth transmission variables included in this analysis, real property unigeniture (transmission of land holdings to a single heir) has the strongest net effect on heritable social class. In contrast, movable property unigeniture has no independent direct effects on social inequality variables, though it does participate in a relationship with class mediated by political succession patterns. These findings support the notion that real property is central to the effect material wealth transmission has on inequality, while movable material property inheritance may serve primarily to support the accumulation and transmission of social wealth $(10 ; 25 ; 26 ; 29)$. We note that unigeniture may be a particularly potent mechanism for concentrating wealth and encouraging formal systems of inequality. We test the same models with intergenerational property 
transmission encoded simply as the presence or absence of any inheritance rules for real and movable property. Because these models result in poorer fit for two of our three inequality variables, and because a parsimonious model offers no improvement on the full model with this data, we retain unigeniture coding and report the alternative implementation of wealth transmission in the supplementary materials.

We also find a direct effect of real property unigeniture on hereditary slavery. Although the magnitude of this effect is smaller than indirect effects of real property inheritance rules on slavery through heritable social class, it serves as further evidence of the importance of real property inheritance in shaping inequality. The use of slave labor as a means of cultivating large parcels of land, which are owned and inherited, explains this relationship to some extent, though it is perhaps surprising that we find no evidence that intensive agriculture participates in this pathway as a driver of real property unigeniture and an indirect driver of the link between real property inheritance patterns and slavery.

Other components of the most parsimonious model also depart from the expected trajectory. The expected stepwise link between resource intensification and wealth transmission occurs in the selected model only in the form of an effect of large domesticated animal keeping on hereditary political succession, and our model predicts that societies that make use of large domesticated animals are, in fact, less likely to have systems of hereditary political succession. If the use of large animals is positively linked to the development of inequality, as Kohler et al. have suggested for agriculturalists (33) and Smith et al. have proposed for pastoralist societies (25), the negative association we find between large animals and transmission of social wealth trends in the opposite 
direction than we might expect for our sample of modern societies. This result also contrasts with prior research that demonstrates positive links between pastoralism, intergenerational of multiple types of wealth, and inequality (31).

Because the data we employ does not distinguish between animals used as a food resource and animals used for labor, we report the results of the same modeling task using a dataset that excludes societies that obtain a majority of their subsistence through pastoralism. The results of this supplementary analysis, which focuses more narrowly on non-pastoral animal husbandry, are qualitatively similar to those reported in Fig. 2, suggesting that the surprising direction of the relationship between large domesticated animals and inequality is not merely an artifact of how animal husbandry has been operationalized. Even in consideration of the limitations of this study, such as the narrow focus on a small subset of the manifestations of social inequality, it is difficult to interpret this apparent contradiction without further, more detailed examination of wealth transmission and inequality in individual pastoralist and agropastoralist societies and in targeted cross-cultural samples.

While intensive subsistence activities might be expected to have primarily indirect impacts on social inequality through positive associations with wealth transmission, we find evidence for stronger direct impacts of subsistence practices on inequality (see Table 1). Though we know that inequality can arise even in the absence of agriculture $(4 ; 10 ; 25 ; 29)$, our results suggest that subsistence activities themselves are important contributors to the social and economic mechanisms out of which rigid inequality structures can arise, independent of wealth transmission patterns that consolidate resources and status for the few. This finding implies that inequality in modern 
societies may arise through multiple pathways, some of which are not dependent on differential accumulation of property and power through wealth transmission practices. Specialization and division of labor in economies associated with intensive agriculture, for example, might create occupation-based stratification in wealth and prestige, regardless of how property or political power are transmitted across generations.

Our failure to perfectly replicate the sequential progression of mechanisms that are thought to have generated early Holocene social inequality might be explainable through alternative, populationfocused theories (34). We might expect a population-driven explanation to be manifested in a trajectory like the one modeled here through effects of population size on wealth transmission or institutionalized social inequality. If the links we find between resource intensification and inequality are artifacts of demographic pressures or the politics of large scale societies, we would expect population size to participate in these pathways. However, we find that population size is linked only to caste, and this association is not particularly strong. While this analysis is not designed to test any particular demographic pressure model in great detail and lacks the statistical power to explore the vast web of cultural, political, and economic pressures at play in its entirety, we find no evidence that suggests our results are driven by the scale of the societies involved.

In addition to testing expectations derived from prior theory, our approach also allows us to examine the independent and interrelated effects of individual observable phenomena associated with the general constructs of environmental conditions, resource intensification, wealth transmission, and institutionalized social inequality. While selecting a parsimonious model of the pathways that link our variables, we are simultaneously able to examine the complexities of these pathways. 
We find, for example, that both real property unigeniture and hereditary political succession are significantly positively associated with heritable social class, but not caste, while movable property unigeniture has no significant direct impacts on any of our institutionalized inequality variables. Furthermore, within the realm of wealth transmission we find evidence that movable property inheritance serves as a link between real property unigeniture and hereditary political succession. This creates a strong indirect effect of real property unigeniture on heritable social class and highlights the complexity of interactions within the domain of wealth transmission.

Our results also point to heritable social class as the measure of institutionalized social inequality that is most strongly associated with the effects of wealth transmission and intensive subsistence practices in modern human cultures. Subsistence and wealth transmission variables are directly and positively associated with heritable social class, while the direct impact of real property unigeniture on slavery is dwarfed by a net indirect effect of real property unigeniture on slavery that is mediated by social class. We find no evidence that caste participates in the pathways linking subsistence or economic defensibility and wealth transmission to inequality. Rather, caste is predicted only by population size in this model. Although this could potentially be interpreted as support for population-based theories or an impact of population size on the development of inequality-reinforcing norms, we propose that this relationship may in fact be an artifact of the highly restricted distribution of caste to societies in a small number of language families in population-dense regions, namely the Indian subcontinent and parts of Africa. The language family random effect in this model is the best predictor of caste, as evidenced by the marginal model fit estimate for this variable (including only fixed effects) of 0.016 , and the conditional fit estimate (including language family and fixed effects) of 0.963 (See supplementary materials). It may be 
difficult to systematically identify the drivers responsible for the origin of caste, given that the caste systems of the world may reflect a very small number of independent origins and a strong signature of shared histories.

\section{Conclusions}

The complex network of effects we identify between environmental, subsistence, inheritance, and social inequality variables suggests that how we measure each of the core cultural constructs associated with a theoretical evolutionary trajectory for social inequality matters to our ability to investigate the processes that create and maintain the institutions that most rigidly support social hierarchies. A priori assumptions might have emphasized intensive agriculture as a means of increasing economic defensibility, and thus a likely predictor of real property inheritance patterns and inequality in turn (35), which could have been tested in a simpler model. However, the inclusion of several variables to represent each component of the theory enables us to identify pathways that deviate from expected trajectories in addition to those that support prior hypotheses.

Our ability to measure the relevant characteristics of societies is limited in practice by the availability of cross-cultural data. With the data used in this analysis we may not be able to capture all of the complexity in the phenomena discussed in great detail in a large body of prior literature, or to capture additional phenomena that may contribute to the modern evolution of inequality. The results presented here do not, in other words, rule out other possible pathways. Many causal relationships have been proposed that may impact individual cultural traits and mediate relationships within this set of variables. For example, though our results do not support the potential importance 
of real property inheritance as a link from agriculture to social inequality, such a pathway might be detectable if specific other variables were included in the analysis. One proposal suggests that metallurgy might arise in agricultural societies and subsequently have an effect on social inequality (36).

There are also numerous other theories about the evolution of slavery (e.g. 37; 38). Due to limitations of data availability we are unable to address every possibility outlined in prior research, and instead focus on whether the influences on contemporary inequality mirror the set of mechanisms for generating inequality that are supported by a large body of literature and distilled in a recent review of that work (10). Because this approach focuses on a specific set of general, society-level phenomena, we urge caution in interpreting results of this type of analysis; our model helps us understand influences on cultural evolution but does not represent a singular, inevitable trajectory for the evolution of institutions of social inequality. Other facets of human culture and behavior are of vital importance in understanding individual systems of slavery, caste, and social class.

Though no analysis can address all hypotheses in light of current data constraints, the results we present here answer several questions and illuminate others that are deserving of further research. For example, the absence of an expected path from intensive agriculture to real property inheritance patterns defies a strong prior expectation and points out a need for further research on the relationship between agricultural practices, land tenure and real property inheritance.

The methods we implement here represent a novel but rigorous way to explore the complex relationships between cultural phenomena that may interact both directly and indirectly, while also 
controlling for shared histories. The structural equation approach illustrated in this analysis makes it possible to examine in detail the empirical evidence for bold theories that have been proposed to explain the evolution of human culture.

\section{Methods}

This study employs Ethnographic Atlas and environmental data for 367 societies available in the D-PLACE database $(11 ; 12 ; 13 ; 14 ; 15 ; 16)$ that are referenced to both specific times and places. This sample of 367 societies is the maximal sample size for which all of the variables included in our study were available. While this sample is distributed around the globe, no special effort was made to control for cultural relatedness through sampling (as in, for example, the Standard CrossCultural Sample). Instead, we control for genealogical relationships explicitly in the design of our mixed effects path models. Although the current lack of a reliable, global cultural phylogeny prevents us from using phylogenetic methods to control for shared histories, we use a random effect of language family in the SEM framework to control for well-established genealogical relationships between societies.

The variables in the study serve as proxies for the more abstract constructs that are central to the hypothesized sequential evolution of early Holocene social inequality, and represent these societies as they were observed at a single point during or near the early 20th century. These data largely result from coding of ethnographic sources, which limits to some extent the ways in which we can test evolutionary hypotheses. For example, the variables selected for this study reflect not only a translation of central theoretical constructs to observable society-level phenomena, but 
also the availability of data describing those phenomena in a large sample of societies around the world. While the data and model we use do not explicitly reconstruct historical states of cultures and their changes over time, the relationships we identify in this empirical data enable inferences about the processes involved in the rise and maintenance of institutionalized social inequality in modern human cultures.

We represent environmental conditions using three variables derived from a principal components analysis (PCA). Although work on early Holocene inequality has characterized the environment largely in terms of temporal trends toward stability immediately prior to that time, the environmental variables included in this analysis allow us to characterize the productivity and predictability of local environments, which are likely to impact the spatial variation we find in human economic activity and cultural norms across a relatively narrow slice of history. Raw data used in this study describe the mean, variance, and predictability of temperature, precipitation, and net primary productivity (NPP) at each location, as well as measures of elevation and slope (see supplementary information). Because this set of environmental variables is known to to be highly correlated, we reduced it to three composite variables through principal components analysis (PCA) to avoid multicollinearity in the downstream SEM analyses (see Table S2). Higher values of PC1 and lower values of PC2 may be expected in productive environments with predictable climates, conditions which may enhance economic defensibility. Higher values of PC3 are associated with topographic complexity, which can increase the patchiness of resources and may thus also contribute to some extent to defensibility. 
ment of inequality (25), and evidence for inequality that predates agriculture suggests that it is also not a necessary condition for the emergence of inequality $(4 ; 29)$. Yet empirical work has found evidence that agriculture - particularly intensive agriculture - and the keeping of large domesticated animals is associated with the wealth distribution and transmission practices that shape social hierarchies $(27 ; 28 ; 33 ; 39)$.

The observed link between subsistence and inequality has been explained as an association between intensive agriculture and property rights, and a resulting concentration of material wealth and political power in agricultural societies (e.g. $19 ; 25 ; 28 ; 39 ; 40)$. The presence of large domesticated animals represents a similar pattern that has been identified for pastoralist societies (31), and also an association between plow agriculture, the maintenance of draught animals, and differential distribution of material wealth (33). We include both the presence of large domesticated animals and the presence of intensive agriculture in our study to represent subsistence activities that have been linked to inequality in prior empirical studies and are likely to impact the economics of resource defense.

We focus on resource intensification as a technological and economic link between environmental conditions and wealth accumulation. While prior research has portrayed this link more broadly as a function of economic defensibility, targeting this mechanism allows this analysis to ask a specific question about the human activities that may have resulted in institutionalized inequality in modern human societies.

Our analysis represents wealth transmission primarily as it relates to material and social wealth. Material wealth transmission is characterized for the purposes of this study by variables 
representing the presence or absence of inheritance rules that bequeath real property (land) and movable property, respectively, to a single heir (unigeniture). Social wealth transmission is characterized here by the presence or absence of hereditary political succession. Although we recognize the importance of embodied wealth in shaping cultures, data limitations prevent us from exploring that component of wealth transmission in this analysis.

Real property has been ascribed a particularly prominent role in differential wealth distribution due to practical limits on its subdivision and productivity $(25 ; 26 ; 41)$. Movable property is considered to be relatively indefensible by Mattison et al. (10), however possession and inheritance of animals has been linked to inequality $(31 ; 33)$. We include both real and movable property to investigate the roles of each in the generation of institutionalized inequality. We hypothesize that these two types of wealth may interact in different ways with intensive agriculture and with the keeping of large animals. Our characterization of material property inheritance in terms of unigeniture reflects an expectation that an inherently unequal pattern of wealth transmission across generations is particularly likely to concentrate resources and power and thus lead to institutionalized inequality. In using a variable that describes hereditary political succession to represent social wealth inheritance, we consider political power to be a reflection of social influence, and its hereditary assignment to be a manifestation of the intergenerational transmission of social wealth.

Persistent, institutionalized inequality, defined clearly by Mattison et al. (10), includes a number of structures of varying levels of formality that emerge in societies to create and maintain stratification. While inequality exists in many forms, at many scales, in many parts of society, it is characterized in this study by the presence or absence of three forms of institutionalized social 
hierarchy that are well described by cross-cultural data: class, caste, and slavery. Each of these variables is encoded in a separate binary (presence/absence) variable, so that relationships between individual types of wealth transmission and specific inequality outcomes may be investigated. For each of these variables, we restrict the presence category to instances where the social stratification system may persist across generations, namely heritable social class, hereditary slavery, and caste. These response variables represent a small subset of the outcomes that can be considered to exemplify persistent, institutionalized, inequality. However, they have the advantages of being reliably identifiable as persistent and institutionalized forms of inequality, of being recoverable for a maximally large, globally distributed sample of societies, and of representing particularly rigid and entrenched mechanisms for enforcing social hierarchies.

Our coding of slavery is complicated by a large number of societies which are coded as "slavery reported but not identified as hereditary or nonhereditary" in the Ethnographic Atlas. We have included these societies in the "hereditary slavery absent" category in the analysis presented here. Results using the alternative binarization (with these societies coded as "hereditary slavery present") differ from the results presented above in having a significant direct effect of intensive agriculture on slavery but no direct effects of $\mathrm{PC} 1$ or real property unigeniture on this outcome. Full results of the analysis including the alternative binarization of the slavery variable are reported in the supplementary materials.

Not all approaches to the evolution of inequality focus on resources, their defense, and the transmission of the resulting wealth. One competing family of theories ascribes the rise of inequality to pressures associated with growing populations and the organization of large-scale societies 
$(18 ; 34 ; 42 ; 43 ; 44)$. Examining the complexities of theories that center on carrying capacity, population pressure, and the roles of individual-level competition and cooperation in creating inequality in detail is beyond the scope of the current analysis of group-level phenomena. However, we are able to incorporate population size into our model and test whether this measure of society size is a significant driver of inequality, as might be predicted by this set of theories. Johnson's scalar stress theory (34), for example, associates hierarchical organization, including social status hierarchies, with the organizational pressures present in larger population. Under such a theory we might expect population size to mediate impacts of agriculture or to serve as an independent driver of inequality. Population size may also impact economic defensibility in complex ways through its effects on within-group coordination and between-group competition (24). Incorporating population size in the model and assessing both its direct and indirect effects on inequality allows us to examine whether resources and population work independently or in concert to impact wealth and social hierarchy, and whether one or the other of these is a more important driver of institutionalized inequality. This variable is encoded as a continuous variable that estimates the number of individuals in each entire ethnic group. More information about the coding of all variables can be found in the supplementary information.

We analyzed the data described above for 367 societies in the R statistical computing environment, using the packages PiecewiseSEM and lme4 for structural equation modeling (45; 46). Language family was included as a random effect to control for potential non-independence of data that may result from common cultural inheritance, following Botero et al. (47). Because no widely accepted global phylogeny of languages or cultures currently exists, we are unable to implement phylogenetic path models, and instead use a less complex but more widely accepted method of 
controlling for historical relationships through the inclusion of well established language family classifications as a random effect in a mixed model framework $(48 ; 49)$

An initial model presents a very simple implementation of a stepwise pathway to inequality, modeled to parallel the trajectory for early Holocene inequality origins outlined by Mattison et al. (10) (Fig. 2b). In this model, environmental conditions are represented by our derived PC variables. These have direct effects only on subsistence (large domesticated animals and intensive agriculture). Subsistence variables represent modern use of resources and technologies to intensify subsistence, and these variables in turn have direct effects only on wealth transmission variables. The three wealth transmission variables have direct effects only on the three social inequality variables. Any relationship between environmental or subsistence variables and inequality can be characterized in this model only by an indirect path through one or more wealth transmission variables.

We may not expect the chain of causal links modeled as a simplistic set of sequential effects in Fig. 2b to serve as the only pathway for inequality to arise, exclusive of any direct impacts of the environment or defensibility-enhancing subsistence practices on inequality. Prior literature presents a more complex picture than the strictly stepwise schema is able to capture, and the trajectory outlined in Fig. 2a does not explicitly rule out additional, direct links. For this reason we also consider a more elaborate model that adheres to the same assumptions about directionality and ordering of causal links, but includes a more complete set of direct paths between variables.

In this second model (Fig. 2c), the directionality of all estimated paths moves from environment to subsistence/population, then inheritance, and finally inequality. Additional paths were 
added to the set in Fig. $2 \mathrm{~b}$ to allow for the possibility of direct effects of predictors on variables farther to the right in the diagram. These direct paths extend from environmental variables to inheritance and social inequality variables, reflecting the possibility that the environment impacts wealth transmission and institutions of inequality independently of agricultural practices and/or population size. Direct paths from agricultural variables and population to social inequality variables are also included. In this model population is treated as an additional potential predictor of wealth transmission and inequality variables, reflecting hypotheses that link inequality to demographic factors and the possibility that resource intensification and society scale have non-independent impacts on inequality outcomes.

Finally, we use the support for individual paths in the full model (Fig. 2c) to develop a more parsimonious model that retains well supported pathways but eliminates unnecessary model complexity (Fig. 3).

\section{Data Availability}

All data are available online at https://d-place.org/. See supplementary materials for detailed information on variables and societies included in this study.

1. Karl, T. L. Economic inequality and democratic instability. Journal of Democracy 11, 149$156(2000)$.

2. Cushing, L., Morello-Frosch, R., Wander, M. \& Pastor, M. The haves, the have-nots, and the health of everyone: The relationship between social inequality and environmental quality. 
Annual Review of Public Health 18, 193-209 (2015).

3. Hurst, C. E., Fitz Gibbon, H. M. \& Nurse, A. M. Social Inequality: Forms, Causes, and Consequences (Routledge, New York, 2017).

4. Hayden, B. Richman, poorman, beggarman, chief: The dynamics of social inequality. In Feinman, G. M. \& Price, T. D. (eds.) Archaeology at the Millennium, 231-272 (Springer, New York, 2001).

5. Bowles, S., Smith, E. A. \& Borgerhoff Mulder, M. The emergence and persistence of inequality in premodern societies. Current Anthropology 51, 7-17 (2010).

6. Flannery, K. \& Marcus, J. The Creation of Inequality (Harvard University Press, Cambridge, MA, 2012).

7. Cashdan, E. A. Egalitarianism among hunters and gatherers. American Anthropologist 82, $116-120$ (1980).

8. Boehm, C. et al. Egalitarian behavior and reverse dominance hierarchy. Current Anthropology 34, 227-254 (1993).

9. Ames, K. M. The archaeology of rank. In Bentley, R. A., Maschner, H. D. \& Chippendale, C. (eds.) Handbook of archaeological theories, 487-513 (AltaMira, Lanham, Maryland, 2007).

10. Mattison, S., Smith, E. A., Shenk, M. K. \& Cochrane, E. E. The evolution of inequality. Evolutionary Anthropology 25, 184-199 (2016).

11. Kirby, K. R. et al. D-PLACE: A global database of cultural, linguistic, and environmental diversity. PLoS One 11, e0158391 (2016). 
12. Murdock, G. P. Ethnographic Atlas (University of Pittsburgh Press, Pittsburgh, 1967).

13. Colwell, R. K. Predictability, constancy, and contingency of periodic phenomena. Ecology 1, $1148-1153$ (1974).

14. Running, S. W., Ramakrishna, N., Glassy, J. M. \& Thornton, P. E. MODIS daily photosynthesis (PSN) and annual net primary production (NPP) product (MOD17) algorithm theoretical basis document (1999). URL http://www.ntsg.umt.edu/modis/ATBD/ATBD_MOD17_v21.pdf. University of Montana, SCF At-Launch Algorithm ATBD Documents.

15. Danielson, J. J. \& Gesch, D. B. Global multi-resolution terrain elevation data 2010 (GMTED2010) (2011). URL http://pubs.usgs.gov/of/2011/1073/pdf/of2011-1073.pdf. U.S. Geological Survey Open-File Report 20111073.

16. Lima-Ribeiro, M. S. et al. Ecoclimate: A database of climate data from multiple models for past, present, and future for macroecologists and biogeographers. Biodiversity Informatics 10, $1-21(2015)$.

17. Johnson, A. W. \& Earle, T. The Evolution of Human Societies (Stanford University Press, Stanford, CA, 2000), 2nd edn.

18. Cohen, M. N. The Food Crisis in Prehistory: Overpopulation and the Origins of Agriculture (Yale University Press, New Haven, 1977).

19. Bowles, S. \& Jung-Kyoo, C. Coevolution of farming and private property during the early 
holocene. Proceedings of the National Academy of Sciences of the United States of America 110, 8830-8835 (2013).

20. Richerson, P. J., Boyd, R. \& Bettinger, R. L. Was agriculture impossible during the pleistocene but mandatory during the holocene? a climate change hypothesis. American Antiquity 66, 387-411 (2001).

21. Boon, J. L. Competition, conflict, and the development of social hierarchies. In Smith, E. A. \& Winterhalder, B. (eds.) Evolutionary Ecology and Human Behavior, 301-337 (Aldine de Gruyter, New York, 1992).

22. Kennett, D. J. \& Winterhalder, B. (eds.) Behavioral ecology and the transition to agriculture (University of California Press, 2006).

23. Dyson-Hudson, R. \& Smith, E. A. Human territoriality: An ecological reassessment. American Anthropologist 80, 21-41 (1978).

24. Chabot-Hanowell, B. \& Smith, E. A. Territorial and nonterritorial routes to power: Reconciling evolutionary ecological, social agency, and historicist approaches. Archaeological Papers of the American Anthropological Association 22, 72-86 (2013).

25. Smith, E. A., Borgerhoff Mulder, B. S., Monique, Gurven, M., Hertz, T. \& Shenk, M. K. Production systems, inheritance, and inequality in premodern societies: Conclusions. Current Anthropology 51, 85-94 (2010).

26. Shennan, S. Property and wealth inequality as cultural niche construction. Philosophical Transactions of the Royal Society B 366, 918-926 (2011). 
27. Borgerhoff Mulder, M. et al. Intergenerational wealth transmission and the dynamics of inequality in small-scale societies. Science 326, 682-688 (2009).

28. Shenk, M. K. et al. Intergenerational wealth transmission among agriculturalists: Foundations of agrarian inequality. Current Anthropology 51, 65-83 (2010).

29. Gurven, M. et al. Domestication alone does not lead to inequality: Intergenerational wealth transmission among horticulturalists. Current Anthropology 51, 49-64 (2010).

30. Kandler, A., Wilder, B. \& Fortunato, L. Inferring individual-level processes from populationlevel patterns in cultural evolution. Royal Society Open Science 4, 170949 (2017).

31. Borgerhoff Mulder, M. et al. Pastoralism and wealth inequality: Revisiting an old question. Current Anthropology 51, 35-48 (2010).

32. Shipley, B. The AIC model selection method applied to path analytic models compared using a d-separation test. Ecology 94, 560-564 (2013).

33. Kohler, T. A. et al. Greater post-Neolithic wealth disparities in Eurasia than in North America and Mesoamerica. Nature 551, 619-622 (2017).

34. Johnson, G. A. Organizational structure and scalar stress. In Renfrew, C., Rowlands, M. J. \& Segraves, B. A. (eds.) Theory and Explanation in Archaeology, 389-421 (Academic Press, New York, 1982).

35. Dye, T. S. Social transformation in old Hawaii: a bottom-up approach. American Antiquity 75, 727-741 (2010). 
36. Peregrine, P. N., Ember, C. R. \& Ember, M. Universal patterns in cultural evolution: An empirical analysis using Guttman scaling. American Anthropologist 106, 145-149 (2004).

37. Pryor, F. L. A comparative study of slave societies. Journal of Comparative Economics 1, 25-49 (1977).

38. Hrnčirir, V. \& Květina, P. Archaeology of slavery from cross-cultural perspective. CrossCultural Research (2017).

39. Price, T. D. Social inequality at the origins of agriculture. In Price, T. D. \& Feinman, G. M. (eds.) Foundations of Social Inequality, 129-151 (Springer, Boston, Massachusetts, 1995).

40. Ember, M., Ember, C. R. \& Russett, B. Inequality and democracy in the anthropological record. In Midlarsky, M. I. (ed.) Inequality, Democracy, and Economic Development, 110130 (Cambridge University Press, Cambridge, 1997).

41. Strassman, B. I. \& Clarke, A. L. Ecological constraints on marriage in rural Ireland. Evolution and Human Behavior 19, 35-55 (1998).

42. Rosenberg, M. Territoriality and sedentism in an evolutionary context. Current Anthropology 39, 653-681 (1998).

43. Turchin, P. \& Gavrilets, S. Evolution of complex hierarchical societies. Social Evolution and History 8, 167-198 (2009).

44. Powers, S. T. \& Lehman, L. An evolutionary model explaining the neolithic transition from egalitarianism to leadership and despotism. Proceedings of the Royal Society B: Biological Sciences 281, 20141349 (2014). 
45. Lefcheck, J. S. piecewiseSEM: Piecewise structural equation modelling in R for ecology, evolution, and systematics. Methods in Ecology and Evolution 7, 573-579 (2015).

46. Bates, D., Mächler, M., Bolker, B. \& Walker, S. Fitting linear mixed-effects models using lme4. Journal of Statistical Software 67, 1-48 (2015).

47. Botero, C. A. et al. The ecology of religious beliefs. Proceedings of the National Academy of Sciences 111, 16784-16789 (2014).

48. Gavin, M. C. et al. The global geography of human subsistence. Royal Society Open Science 5 (2018).

49. Roberts, S. G., Winters, J. \& Chen, K. Future tense and economic decisions: Controlling for cultural evolution. PLoS ONE 10 (2015).

Acknowledgements The authors would like to thank Heidi Colleran for feedback which improved the paper. The work presented in this paper is supported by the National Science Foundation under grant no. 1519987. The funders had no role in study design, data collection and analysis, decision to publish, or preparation of the manuscript. 